\title{
Method for determination of the absorption coefficient in films based on photoluminophore suspension for white LEDs
}

\author{
D.N. Khmil, A.M. Kamuz, P.F. Oleksenko, V.G. Kamuz, N.G. Aleksenko, O.A. Kamuz \\ V. Lashkaryov Institute of Semiconductor Physics, NAS of Ukraine, \\ 41, prospect Nauky, 03680 Kyiv, Ukraine. \\ Phone: +38 (044) 525-6168; e-mail: deniskhmil@ukr.net
}

\begin{abstract}
Developed in this work is the method for measuring the absorption coefficient in optically non-homogeneous media (films prepared from photoluminophore suspension). Using this new method, the authors have measured the absorption spectrum of the above films with inorganic photoluminophore FLY-7. At the film absorption peak (near $448 \mathrm{~nm}$ ) and photoluminophore concentration close to $20 \%$, the absorption coefficient reaches $124 \mathrm{~cm}^{-1}$. Ascertained have been the conditions that should be provided when using this method.
\end{abstract}

Keywords: absorption coefficient, photoluminophore, light scattering, white LED.

Manuscript received 18.12.14; revised version received 25.03.15; accepted for publication 27.05.15; published online 08.06.15.

\section{Introduction}

Nowadays, LED lighting becomes widely spread as one of promising energy-saving technologies. In lab conditions, these light sources reach the values of efficiency up to $303 \mathrm{Lm} / \mathrm{W}$ for the correlated color temperature $5150 \mathrm{~K}$ [1]. Due to this efficiency, it becomes possible to change not only incandescent filament lamps but the compact luminescent ones, too. However, beside their high efficiency, they should satisfy some other requirements: to have the correlated color temperature within the range 2700 to $6500 \mathrm{~K}$, high index of color transfer and be resistant to degradation.

These parameters depend predominantly on the layer of photoluminophore suspension, i.e., on photoluminophore properties. In relation with it, development and synthesis of new highly-efficient photoluminophores last, which means necessity to satisfy specific requirements to measurements of their optical and colorimetric parameters. Among optical parameters, the absorption coefficient plays a main role, because it defines the quantum yield and, respectively, the efficiency of white LEDs.
Up to date, the only several methods for determining the absorptive of photoluminophore suspension are known. One of the most used methods is the measurement of excitation spectrum (i.e., dependence of the luminescence intensity corresponding to the wavelength of the luminescent peak on the excitation wavelength). As it was shown in the works $[5,6]$, the luminescence intensity at the wavelength $\lambda_{\max }$ is in proportion to the absorption coefficient only in specific conditions:

- low extinction of light that passes through the studied sample (it means using thin luminescent samples);

- $\quad$ absence of saturation in matter that radiates (i.e., one should use low light fluxes for excitation);

- the same light intensity that serves for excitation within the whole studied range of wavelengths;

- $\quad$ independency of the luminescence yield of the light wavelength that provides excitation over the whole range.

In practice, these conditions are never fulfilled. As a result, this method is capable to provide only qualitative data. Especially often, the first condition is 
not fulfilled, since there always used are infinitely thick samples but not the thin ones. In this case, if the conditions 2 and 3 are fulfilled, the brightness of luminescence that arises under action of beams with a definite wavelength is in proportion to the luminescence yield but not to the absorption coefficient value $[2,3]$.

Recently, there appear the works where the methods of modeling of light propagation in a layer of photoluminophore suspension are used. Proposed in the works $[7,8]$ have been complex methods for calculating the absorption coefficient value only at the wavelength alone (455 $\mathrm{nm}[8])$.

The aim of this work was in development of a new method for express determining the dependence of the absorption coefficient on the wavelength of optically inhomogeneous films prepared from photoluminophore suspension.

\section{Theory}

As it is known from classical optics, the absorption coefficient for a plane-parallel light beam is determined via changes of the light intensity caused by absorption in optically homogeneous medium (Bouguer-Lambert law). It is the so-called natural absorption coefficient. But in optically inhomogeneous medium, the light intensity is changed not only by absorption but due to light scattering by optical inhomogeneities (to be more exact, due to transformation of the scattering indicatrix) [3-6].

In the theory of multiple scattering by W. Hartel $[5,6]$ that is based on the Mie theory, it was shown how the light plane-parallel beam changes its scattering indicatrix up to the spherical shape when propagating in optically inhomogeneous medium.

Let us consider in more details the process of transformation of the scattering indicatrix shape for the case of blue LED light $(456 \mathrm{~nm})$ passage through a composite film prepared from photoluminophore suspension, when using the optical setup shown in Fig. 1.

Let us assume that a quasi-plane wave is incident onto the film, and its scattering indicatrix at the input to optically inhomogeneous medium has the shape of a narrow peak (Fig. 2a, curve 1), and the space angular aperture $\Omega$ of the radiation source is equal to that of the detector in a spectroradiometer (for example, for the device HAAS-2000 $\Omega$ is close to 0.02391 steradian). We shall not take into account the fine structure of the indicatrix describing the light scattering by microparticles.

At first, let us consider the case when microparticles do not absorb light but only scatter it. Let the light intensity in the plane 1 is equal to $I_{1}$ (Fig. 2b). When passing from the plane 1 to the plane 2, light undergoes multiple scattering. As a result, the scattering indicatrix will be expanded (Fig. 2a, curve 2), and the light intensity will be decreased along the direction of the coordinate axis $L$ down to the value $I_{2}$ wa $(F i g .2 \mathrm{c}$, curve 1). The subscript 'wa' means "without absorption", i.e., microparticles of powder-like photoluminophore do not absorb light but only scatter it.

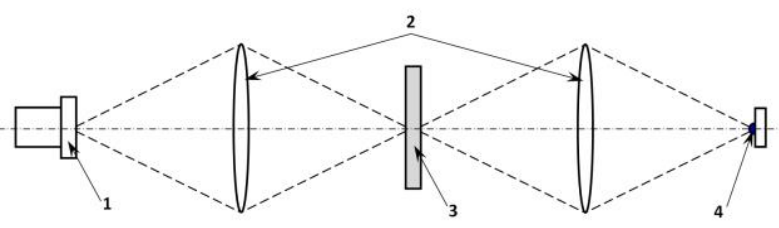

Fig. 1. One of the possible setups for studying the absorption spectra: 1 - detector, 2 - lenses, 3 - studied sample, 4 - light source (LED, $456 \mathrm{~nm})$.

On its path from the plane 2 to the plane 3 , the shape of the scattering indicatrix continues to change, and in the plane 3 it becomes practically spherical (Fig. 2a, curve 3), while the light intensity along the direction of the $L$ axis decreases down to the value $I_{3}$ wa (Fig. 2c, curve 1).

In the plane 4, the indicatrix of light scattering acquires the spherical shape, and the light intensity $I_{4}$ wa possesses equal values in an arbitrary direction within the space angle $4 \pi$ (Fig. 2a, curve 4 , Fig. 2c, curve 1 ).

The range of the film (between the planes 1 and 4), where transformation of the shape inherent to the indicatrix of light scattering takes place, we shall call the "range of transformation", and its thickness we shall designate as $l_{\text {tr }}$. It should be noted that this parameter depends on composition of photoluminophore suspension, concentration of photoluminophore powder in the film, its thickness, and the refractive indexes of powder and substance that is used as a binder.

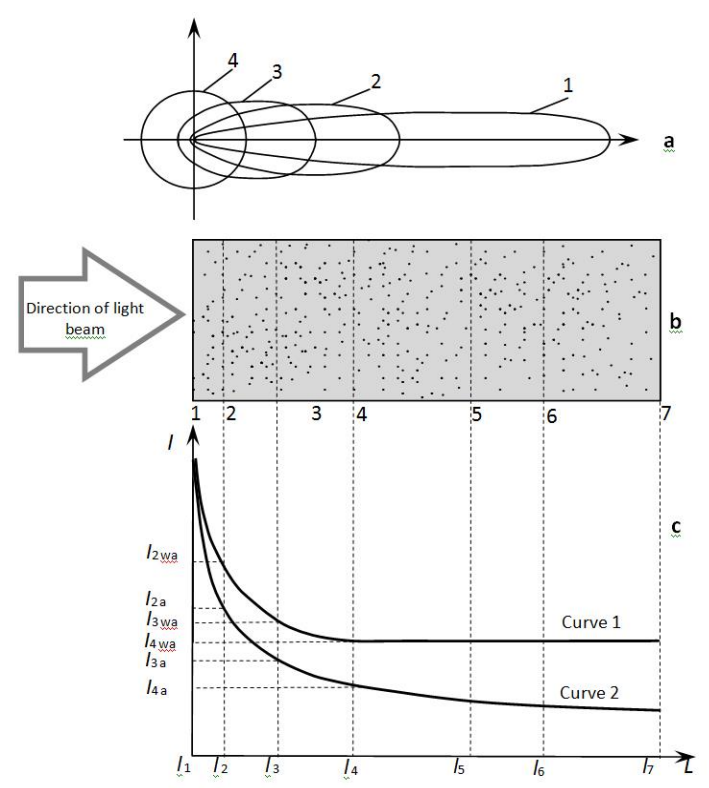

Fig. 2. Transformation of the shape inherent to the light indicatrix within the range $1-4(a)$, schematic view of the film prepared from photoluminophore suspension $(b)$, and the dependences of the light intensity on the film thickness $L$ for microparticles without light absorption (1) and those absorbing light (2) (c). 
As in the planes 5,6 and so on, the indicatrixes of light scattering also will have the spherical shape, and particles of powder-like photoluminophore do not absorb light, the light intensity along the $L$ axis in these planes should have some constant value, i.e., $I_{5 \mathrm{wa}}=I_{6 \mathrm{wa}}=I_{7 \mathrm{wa}}$ $=I_{4}$ wa $($ Fig. 2b, horizontal part of the curve 1$)$. Thus, due to transformation of the scattering indicatrix, the light intensity along the $L$-axis decrease in transformation region even under the absence of light absorption by particles of powder-like photoluminophore (Fig. 2c, the part $1-4$ of the curve 1 ).

In what follows, we shall assume that the relative elementary change of the light intensity $\frac{d I(l)}{I(l)}$ along the $L$-axis in every elementary layer $d l$ in the film does not depend on the light intensity and is in proportion to the thickness of the elementary layer, that is

$$
\frac{d I(l)}{I(l)}=-\alpha_{\mathrm{tr}} d l
$$

where $\alpha_{\text {tr }}$ is the coefficient of proportionality or coefficient of transformation of the scattering indicatrix shape.

The total change in the light intensity (along the Laxis) in the transformation region can be found after integrating the left and right parts from $I_{0}$ to $I_{1}$ and the film thickness 0 up to $l\left(0<l<l_{4}\right)$ :

$$
\int_{I_{0}}^{I_{l}} \frac{d I(l)}{I(l)}=-\int_{0}^{l_{4}} \alpha_{\mathrm{tr}} d l .
$$

As a result, one can obtain

$$
\ln \left(\frac{d I(l)}{I(l)}\right)=\ln \left(-\alpha_{\mathrm{tr}} l\right)
$$

or

$$
I(l)=I_{0} \cdot e^{-\alpha_{\mathrm{tr}} \cdot l}
$$

where $I_{0}$ is the intensity of light beam at the input to the film, $l$ - thickness of the substance layer through which light passes.

Changes in the shape of scattering indicatrix take place only within the transformation region (from $l=0$ up to $l=l_{4}$ ).

The parameter of transformation region, which defines the velocity of changes in the light intensity, is the transformation coefficient for the shape of scattering indicatrix $\alpha_{\text {tr }}$.

When photoluminophore micro particles absorb light, the light intensity in the transformation region (Fig. 3c, part of the film 1-4, curve 2) decreases both due to scattering indicatrix transformation and absorption, while on the part 4-7 only due to absorption (Fig. 3c, curve 2). At the planes 2, 3, 4, 5, 6 and 7, the light intensity will have the following values $I_{2 \mathrm{a}}, I_{3 \mathrm{a}}, I_{4 \mathrm{a}}$, $I_{5 \mathrm{a}}, \quad I_{6 \mathrm{a}}$ and $I_{7 \mathrm{a}}$, respectively (index 'a' means that photoluminophore microparticles absorb light).

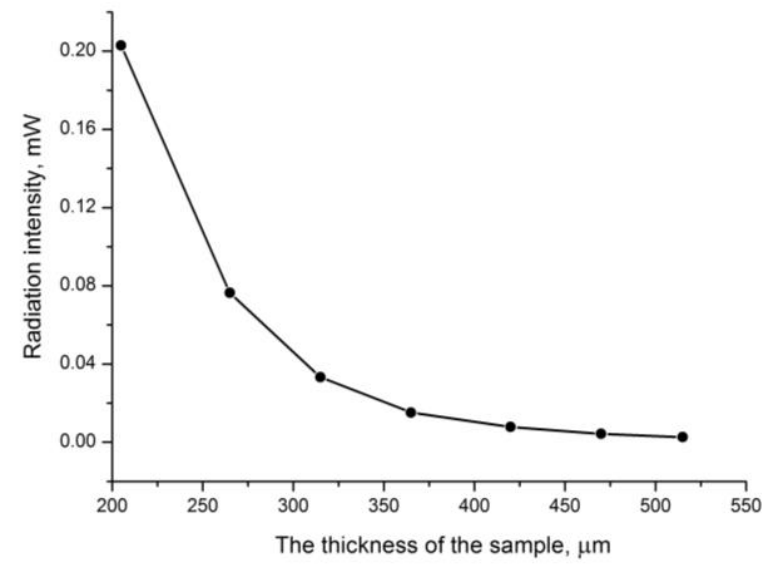

Fig. 3. Dependence of the transmitted radiation intensity on the thickness of the film prepared from composite mixture. The light wavelength is $456 \mathrm{~nm}$.

As it is well known from classical optics, to determine the absorption coefficient of optically homogeneous media, one usually uses the BouguerLambert law

$I(l)=I_{0} e^{-\alpha_{\lambda} \cdot l}$,

where $\alpha_{\lambda}-$ absorption coefficient.

So, for the parts 1-4 and 4-7, with account of the transformation coefficient for the scattering indicatrix, the Bouguer-Lambert formulae acquire the following appearance:

$I(l)=I_{0} \cdot e^{-\left(\alpha_{\mathrm{tr}}+\alpha_{\lambda}\right) \cdot l}$ (from $l=0$ up to $\left.l<l_{4}\right)$

and

$I(l)=I\left(l_{4}\right) \cdot e^{-\alpha_{\lambda} \cdot l}=I_{0} \cdot e^{-\left(\alpha_{\mathrm{tr}}+\alpha_{\lambda}\right) \cdot l_{4}} \cdot e^{-\alpha_{\lambda} \cdot l}$

(from $l=l_{4}$ up to $l=l_{7}$ ),

where $I\left(l_{4}\right)$ is the light intensity after passing through the layer with the thickness $l_{4}, I(l)$ - light intensity in the part $1-4$ and $4-7$, respectively.

Using the same law, expressions for the light intensity at the planes 5 and 7 that are out of the transformation range can be written, respectively, as follows:

$I\left(l_{5}\right)=I_{0} \cdot e^{-\left(\alpha_{\mathrm{tr}}+\alpha_{\lambda}\right) \cdot l_{5}}=I_{0} \cdot e^{-\left(\alpha_{\mathrm{tr}}+\alpha_{\lambda}\right) \cdot l_{4}} \cdot e^{-\alpha_{\lambda}\left(l_{5}-l_{4}\right)}$

and

$I\left(l_{7}\right)=I_{0} \cdot e^{-\left(\alpha_{\mathrm{tr}}+\alpha_{\lambda}\right) \cdot l_{7}}=I_{0} \cdot e^{-\left(\alpha_{\mathrm{tr}}+\alpha_{\lambda}\right) \cdot l_{4}} \cdot e^{-\alpha_{\lambda}\left(l_{7}-l_{4}\right)}$.

Dividing Exp. (9) by (10), one can obtain

$$
\begin{aligned}
& \frac{I\left(l_{5}\right)}{I\left(l_{7}\right)}=\frac{I_{0} \cdot e^{-\left(\alpha_{\operatorname{tr}}+\alpha_{\lambda}\right) \cdot l_{4}} \cdot e^{-\alpha_{\lambda}\left(l_{5}-l_{4}\right)}}{I_{0} \cdot e^{-\left(\alpha_{\operatorname{tr}}+\alpha_{\lambda}\right) \cdot l_{4}} \cdot e^{-\alpha_{\lambda}\left(l_{7}-l_{4}\right)}}=\frac{e^{-\alpha_{\lambda}\left(l_{5}-l_{4}\right)}}{e^{-\alpha_{\lambda}\left(l_{7}-l_{4}\right)}}= \\
& =\frac{e^{-\alpha_{\lambda} \cdot l_{5}} \cdot e^{\alpha_{\lambda} \cdot l_{4}}}{e^{-\alpha_{\lambda} \cdot l_{7}} \cdot e^{\alpha_{\lambda} \cdot l_{4}}}=e^{-\alpha_{\lambda}\left(l_{5}-l_{7}\right)} .
\end{aligned}
$$


Let us take the logarithm of Exp. (11) and write the expression for the absorption coefficient of the suspension film $\alpha_{\lambda}$ :

$\alpha_{\lambda}=\frac{\ln \frac{I\left(l_{5}\right)}{I\left(l_{7}\right)}}{\left(l_{7}-l_{5}\right)}$.

It is seen from Exp. (12) that, to find the value of absorption coefficient inherent to the suspension film, it is necessary to determine only the light intensities $I\left(l_{5}\right)$ and $I\left(l_{7}\right)$ at the outputs of films with the thicknesses $l_{5}$ and $l_{7}$, respectively. It means that one should use two composite films with the thicknesses higher than the transformation region. Therefore, this method for measuring the absorption coefficient inherent to the suspension film we called "the method of two thicknesses". It should be noted that our method differs from the classical method of two thicknesses that is often used when determining the absorption coefficient of optically homogeneous medium. If using our method, it is necessary that every thickness should exceed the thickness of scattering indicatrix transformation in this film. But it implies that first one should determine the thickness of indicatrix transformation range.

\section{Experiment and results}

The developed method needs knowledge of the thickness inherent to the region of indicatrix transformation in the composite film. It is obvious that its value depends on the concentration of photoluminophore microcrystals in binder, sizes of these microcrystals, refractive indexes of photoluminophore microparticles and binder.

The simplest way to determine the thickness of transformation range is to plot the dependence the radiation intensity on the thickness of composite film (Fig. 3) after measurements using the optical scheme shown in Fig. 1. To determine the thickness of transformation range with the method of two thicknesses, we used the 205- and 515-nm thick films. As seen from Fig. 3, the thickness of transformation region is approximately $350 \mu \mathrm{m}$. Thereof, for the method to be applicable, it is expedient to use films with the thickness higher than $350 \mu \mathrm{m}$.

To determine the absorption coefficient of composite films prepared from photoluminophore suspension, we made these films with various thicknesses $(205,265,315,365,420,470$, and $515 \mu \mathrm{m})$. Composition of the films consisted of: epoxy resin, photoluminophore FLY-7 (20\%) and thixotropic dopants. As an excitation source, we used a blue LED with the emission peak at the wavelength $456 \mathrm{~nm}$. These investigations were performed using the scheme shown in Fig. 1.

Fig. 4 shows absorption spectra obtained using the method of two thicknesses $(8-10)$ and classical
Bouguer-Lambert law (curves 1-7, without account of $\alpha_{\text {tr }}$ value). Having analyzed the obtained data, one can draw the conclusion that, when using the BouguerLambert law deduced for a parallel light beam and optically homogeneous medium, the obtained data are unreliable. So, when using the sample with the thickness $205 \mathrm{~nm}$ (Fig. 4), the curve 1 mainly describes a decrease in the light intensity caused by transformation of the scattering indicatrix. When the sample thickness is increased, the influence of this transformation is gradually decreased, while contribution of photoluminophore absorption is increased. It results in appearance of the absorption peak at the wavelength $448 \mathrm{~nm}$ (in Fig. 4, the curves correspond to the following sample thicknesses: $2-265,3-315,4-365,5-420$, $6-470$, and $7-515 \mu \mathrm{m})$.

When applying the method of two thicknesses (sample thicknesses being chosen to exceed the thickness of transformation range), we obtained the absorption spectra (Fig. 4, curves 8-10). To plot the curve 8 , we used the samples with the thicknesses 365 and $415 \mu \mathrm{m}$, curve $9-365$ and $470 \mu \mathrm{m}$, and curve $10-$ 365 and $515 \mu \mathrm{m}$.

The accuracy of this method, like to that inherent to the Bouguer-Lambert formula, is defined by the accuracy of sample thickness measurements as well as by homogeneity of photoluminophore distribution, and by the influence of sample luminescence. The latter increases the absorption coefficient value with increasing the thickness. These reasons enable to explain insignificant differences between the curves 8 to 10 in Fig. 4. To enhance the accuracy of the method, one should use more samples with different thicknesses (higher than the transformation one) as well as apply the excitation source with the spectral half width lower than the Stokes shift inherent to this photoluminophore.

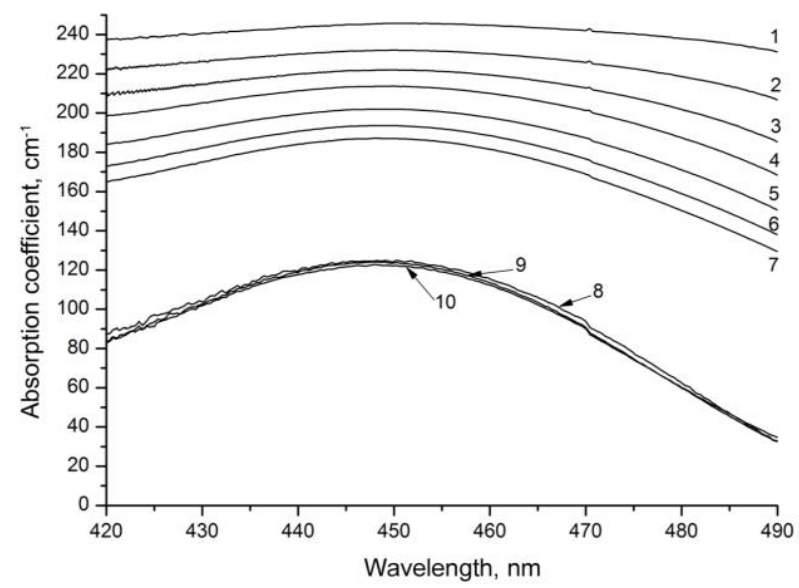

Fig. 4. Dependence of absorption coefficients on the light wavelength after determination by using the Bouguer-Lambert law $(1-7)$ or the method of two thicknesses $(8-10)$. 


\section{Conclusions}

- The results of this work have demonstrated that the shape of scattering indicatrix in optically inhomogeneous medium is changed from narrow directed up to the spherical one. It has been shown that, for low thicknesses of composite films and low concentrations of microparticles, the change in emission intensity is mainly realized via the change in the scattering indicatrix. Only when the film thickness exceeds that for the range scattering indicatrix transformation, the change in the emission intensity is exclusively conditioned by absorption.

- Performed in this work has been investigation of changes in the emission intensity in optically inhomogeneous medium (film prepared from photoluminophore suspension) in dependence of the film thickness.

- Having taken into account the change in the scattering indicatrix, we offer the new method of two thicknesses for determination of the absorption coefficient dependence of composite films on the light wavelength and consider the conditions that should be kept when using it.

- It has been shown experimentally that, when using the classical Bouguer-Lambert law for determination the spectral dependence of the absorption coefficient, it is necessary to take into account transformation of light scattering indicatrix.

- The developed method has been demonstrated using inorganic photoluminophore FLY-7 with the mean size of microcrystals $5 \mu \mathrm{m}$. Near the absorption peak of the film (448 nm), its absorption coefficient reaches the value $124 \mathrm{~cm}^{-1}$, if the photoluminophore concentration is close to $20 \%$.

\section{References}

1. http://www.cree.com/News-and-Events/CreeNews/Press-Releases/2014/March/300LPW-LEDbarrier

2. D.N. Khmil, A.M. Kamuz, P.F. Oleksenko, V.G. Kamuz, N.G. Aleksenko, O.A. Kamuz, Luminophors in white LEDs: advantages and deficiencies // Optoelektronika $i$ poluprovodnikovaya tekhnika, 47, p. 5-23 (2012), in Russian.

3. V.L. Lyovshin, Photoluminescence of Liquids and Solids. Gostekhizdat, Moscow-Leningrad, 1951, p. 39-44 (in Russian).

4. A. Isimaru, Propagation and Scattering of Waves in Randomly-Inhomogeneous Media. Vol. 2. Moscow, Mir, 1981, p. 20-26 (in Russian).

5. T.H. James, Theory of Photographic Process. Leningrad, Khimiya, 1980, p.565-570 (in Russian).

6. H.H. Theissing, Macrodistribution of light scattered by dispersions of spherical dielectric particles // JOSA, 40, p. 232-242 (1950).

7. D.-Y. Kang, E. Wu, and D.-M. Wang, Modeling white light-emitting diodes with phosphor layers // Appl. Phys. Lett. 89, 231102 (2006).

8. Z. Liu, S. Liu, K. Wang, and X. Luo, Measurement and numerical studies of optical properties of YAG:Ce phosphor for white light-emitting diode packaging // Appl. Opt. 49, p. 247-257 (2010). 